\title{
Anelastic to Plastic Transition in Metallic Glass-Forming Liquids
}

\author{
John S. Harmon, ${ }^{1}$ Marios D. Demetriou, ${ }^{1, *}$ William L. Johnson, ${ }^{1}$ and Konrad Samwer ${ }^{2}$ \\ ${ }^{1}$ Keck Engineering Laboratories, California Institute of Technology, Pasadena, California 91125, USA \\ ${ }^{2}$ I. Physik Institute, University of Goettingen, Goettingen, Germany
}

(Received 8 June 2007; published 28 September 2007)

\begin{abstract}
The configurational properties associated with the transition from anelasticity to plasticity in a transiently deforming metallic glass-forming liquid are studied. The data reveal that the underlying transition kinetics for flow can be separated into reversible and irreversible configurational hopping across the liquid energy landscape, identified with $\beta$ and $\alpha$ relaxation processes, respectively. A critical stress characterizing the transition is recognized as an effective Eshelby "backstress," revealing a link between the apparent anelasticity and the "confinement stress" of the elastic matrix surrounding the plastic core of a shear transformation zone.
\end{abstract}

DOI: 10.1103/PhysRevLett.99.135502

Some of the earliest efforts to describe the mechanics of deformation and flow of metallic glasses and liquids were carried out by Argon [1]. Inspired by the deformation of soap bubble rafts [2], Argon proposed that these materials deform by plastic rearrangements of atomic regions involving tens of atoms, termed shear transformation zones (STZ's). Argon further recognized that these plastically rearranging regions were not free but confined within an elastic medium [3]. Using Eshelby's insightful analysis [4], he estimated the effect of elastic matrix confinement on the energy barrier separating the initial and transformed state of the STZ. He further argued that the elastic matrix confinement of an isolated transformed STZ would lead to reversible elastic energy storage in the STZ-matrix system, implying that transformed STZ's have a memory of their original untransformed state. Interestingly, Argon's concept was recently studied in a deforming metallic glass foam, where buckled membranes and their accommodating stress fields were observed to behave like elastically confined STZ's [5].

As recognized by Johari and Goldstein [6], the underlying relaxation mechanisms of liquids and glasses are governed by two kinetic processes: a fast process, termed the $\beta$ process, viewed as a locally initiated and reversible process, and a slow process, termed the $\alpha$ process, viewed as a large scale irreversible rearrangement of the material. From a potential energy landscape perspective, Debenedetti and Stillinger [7] have identified the $\beta$ transitions as stochastically activated hopping events across "subbasins" confined within the inherent "megabasin" (intrabasin hopping) and the $\alpha$ transitions as irreversible hopping events extending across different landscape megabasins (interbasin hopping). A 1D section of a potential energy landscape illustrating this concept is presented schematically in Fig. 1. Johnson and Samwer [8] have recently shown that, by employing a sinusoidal function to describe the megabasin potential energy density, a scaling law for the yield strength of a frozen-in configuration arises in terms of the curvature of the potential energy density function (i.e., the isoconfigurational shear modulus), re-
PACS numbers: 61.43.Dq, 62.10.+s, 62.20.Dc, 62.40.+i

vealing a universal shear strain limit of $\gamma_{c}=0.036$. More recently, Demetriou et al. [9-11] extended this sinusoidal potential to describe the average interbasin configurational hopping arriving at accurate Newtonian and nonNewtonian viscosity laws. In the present study, we seek to explore the relevance of the underlying configurational hopping mechanisms to Argon's concept of "dressed" STZ's. We argue that isolated STZ transitions confined within the elastic matrix are associated with the faster $\beta$ processes, while percolation of these transitions leading to

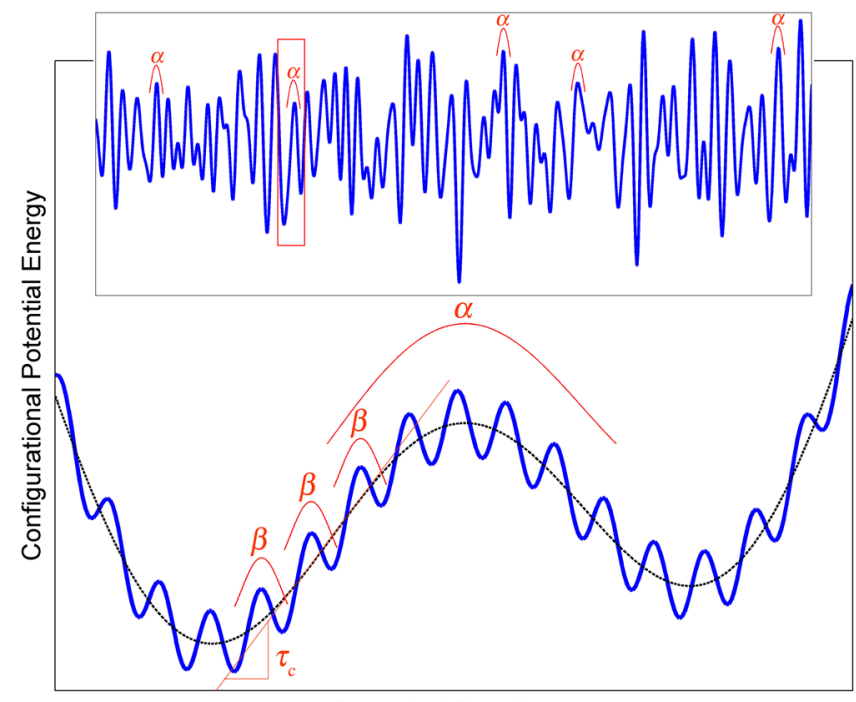

Canonical Coordinate

FIG. 1 (color online). Schematic of a 1D section of a potential energy landscape (projected onto an average shear strain coordinate) in the vicinity of a megabasin (as proposed in Ref. [7]). Anelastic reversible $\beta$ (intrabasin) as well as plastic irreversible $\alpha$ (interbasin) hopping events are shown. The average slope of the potential energy density function, which is taken here to represent the STZ confinement stress, is also indicated. Inset: Schematic of a 1D landscape morphology extending across multiple random megabasins. Plastic irreversible $\alpha$ relaxation (interbasin) hopping events are shown. A representative megabasin is highlighted. 
the collapse of the confining matrix and breakdown of elasticity are associated with the slower $\alpha$ process. We investigate these transitions by probing the instantaneous configurational properties via mechanical, calorimetric, ultrasonic, and strain recovery experiments.

For the experiments, we utilized amorphous $\mathrm{Pd}_{43} \mathrm{Ni}_{10} \mathrm{Cu}_{27} \mathrm{P}_{20}$ specimens that were annealed and structurally relaxed to a reference state at $548 \mathrm{~K}(21 \mathrm{~K}$ below the calorimetric glass transition temperature $T_{g}$ ) for 48 hours prior to testing. Constant strain-rate compression experiments at $548 \mathrm{~K}$ and $1 \times 10^{-4} \mathrm{~s}^{-1}$ strain rate were performed using the setup described in Ref. [12]. At that temperature and strain rate, the biasing rate is comparable to the configurational relaxation rate, and, consequently, the material can relax stress by undergoing homogeneous liquidlike inelastic deformation rather than deforming heterogeneously (by localized shear banding) like an out-ofequilibrium glass. Thus, at the chosen temperature and strain rate, the material can be properly termed a "liquid." The thermodynamic properties associated with the instantaneous configurational state of the material during transient deformation were measured ex situ, i.e., after the specimens were unloaded and quenched to room temperature at a quench rate sufficient to capture the instantaneous liquid configuration. The transient deformation response was thus probed by deforming different specimens to different total strains followed by rapid quenching. The inelastic strain (total strain minus the elastic strain) at each transient stage was evaluated by accurately measuring the specimen height change. The elastic softening induced by deformation at each transient stage was assessed by measuring the high-frequency isoconfigurational shear modulus $G$. Room temperature shear moduli were evaluated ultrasonically using the setup and method described in Ref. [13]. The isoconfigurational shear moduli at the experiment temperature were then estimated by extrapolating the room temperature measurements using a measured linear Debye-Grüneisen constant of $15 \mathrm{MPa} / \mathrm{K}$ [14]. The change in configurational potential energy at each transient stage was evaluated by measuring the change in recovered enthalpy $\Delta h$. Stored enthalpies were recovered by reheating the deformed specimens through the glass transition using differential scanning calorimetry, and changes referenced to the stored enthalpy of a specimen relaxed at $548 \mathrm{~K}$ with no imposed deformation were measured (see [15]). Finally, the ability of the material to undergo anelastic recovery at each transient stage was assessed by measuring the fraction of inelastic strain recovered upon subsequent annealing near $T_{g}$ (see [16]).

In Fig. 2, we present the stress-strain responses of specimens deformed to total uniaxial strains ranging from 0.0102 to 0.211 . As established previously [12], these liquids accommodate stress by undergoing an overshooting stress-strain response associated with a peak stress $\sigma_{c}$ and, ultimately, at a strain of $\sim 0.1$ stabilize at a steady flow stress state. As argued previously [15], this uniaxial strain

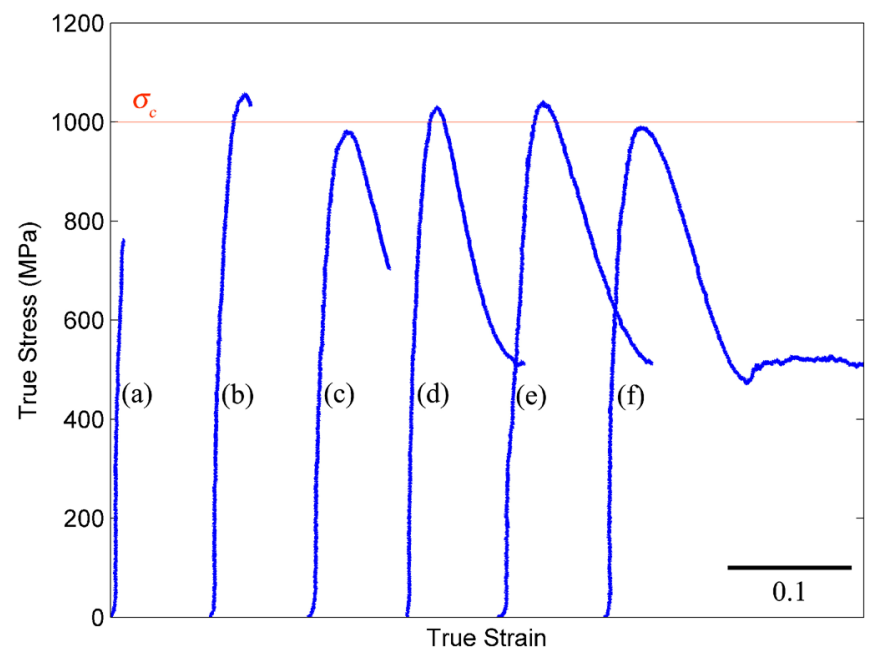

FIG. 2 (color online). Stress-strain responses of specimens deformed to different strains. The uniaxial total strains are (a) 0.0102 , (b) 0.0335 , (c) 0.0655 , (d) 0.0952 , (e) 0.1185 , and (f) 0.2110. The uniaxial inelastic strains are (a) 0.0028, (b) 0.0196, (c) 0.0541, (d) 0.0858, (e) 0.1106, and (f) 0.2042 . An approximate value for the critical stress $\sigma_{c}$ is indicated.

to steady state of $\sim 0.1$, which roughly corresponds to an inelastic shear strain of 0.14 , is observed to be universal for metallic glass-forming liquids and coincides with the crossing of megabasins whose average configurational spacing is estimated to be $4 \gamma_{c}=4 \times 0.036 \approx 0.14$ [8]. This typical stress-strain response is also evident in the present sequential tests shown in Fig. 2, which we interpret as follows: The initial rising stress response reflects a subcritical activated state of the system dominated by intrabasin hopping; the peak stress state represents a critically activated state of the system at which interbasin hopping becomes probable; the decreasing stress-strain response reflects a postcritical activated state of the system dominated by interbasin hopping; the eventual steady stress state reflects a kinetic balance between the biasing rate and the interbasin hopping rate. We now validate this proposal by investigating the configurational properties of the material at the different stages of transient deformation.

In Fig. 3, we plot the calorimetrically measured recovered enthalpy $\Delta h$ (which at ambient pressure we take to represent the stored configurational potential energy) against the inelastic strain. Within the transient deformation regime, $\Delta h$ is found to increase monotonically; however, as deformation reaches steady state, $\Delta h$ stabilizes. By integrating the stress-strain curves and subtracting the elastic energy (determined from the applied stress and measured elastic constants), we estimate the inelastic input mechanical work at each transient deformation stage. Plotting $\Delta h$ against the estimated inelastic mechanical work (inset in Fig. 3), a nearly one-to-one correspondence is found within the transient regime, implying that metallic glass-forming liquids are very efficient in storing input mechanical energy during transient deformation. As steady state is attained, the stored energy saturates, and a dissipat- 


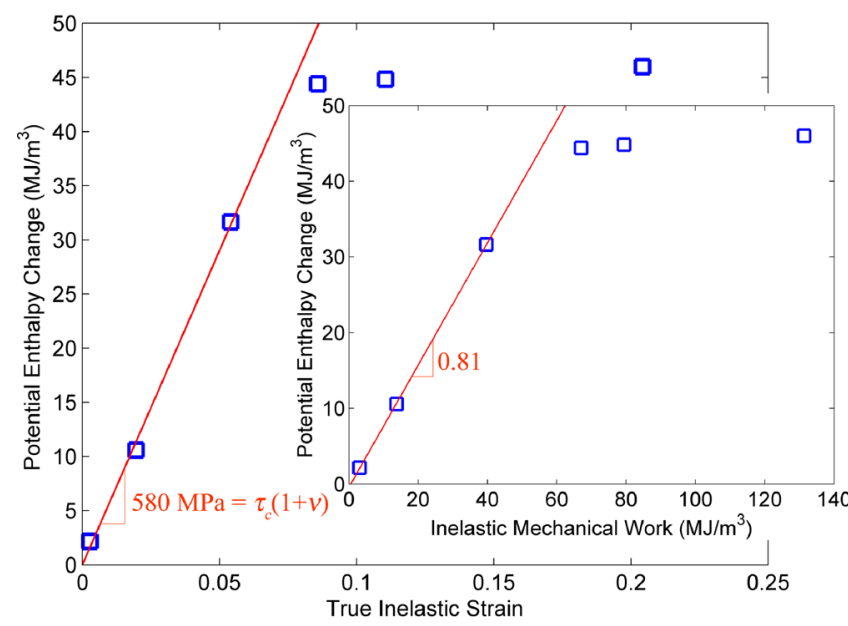

FIG. 3 (color online). Calorimetric measurements of the recovered enthalpy $\Delta h$ plotted against the measured inelastic strain. Inset: $\Delta h$ plotted against the estimated inelastic mechanical work. The lines represent linear regressions to the data within the transient deformation regime.

ing process takes over. The storage of configurational energy upon transient deformation indicates a growing capability of the system to stochastically attempt increasingly higher-energy barrier crossing events within its inherent megabasin. The plateau in configurational potential energy storage attained in steady state is a consequence of the system gaining enough energy to deterministically escape its megabasin and irreversibly accommodate the stress bias and input energy by exploring different configurational states of the liquid landscape.

In Fig. 4, we plot the ultrasonically measured $G$ against the measured inelastic strain. Within the transient regime, $G$ decreases monotonically, while at steady state $G$ stabilizes. The existence of a steady-state high-frequency shear modulus suggests that $G$ is a thermodynamic property of the material associated with the configurational energy state defined by temperature and strain rate. Indeed, $G$ has previously been shown to be uniquely and reversibly dependent on temperature $[10,11,13]$ as well as strain rate [9,11]. More precisely, $G$ was found to be a unique decreasing function of $\Delta h$ independent of whether $\Delta h$ is induced by thermal excitation or mechanical deformation $[11,15]$. This decreasing dependence of $G$ on $\Delta h$ reflects the decreasing curvature of the potential energy density function with increasing configurational energy and has been validated computationally [17]. In the inset in Fig. 4, we plot the transient $G$ data against the transient $\Delta h$ data gathered here, superimposed on steady-state $G$ vs $\Delta h$ data gathered previously at $548 \mathrm{~K}$ for different strain rates [15]. As seen in the plot, the transient $G$ data obey the same functional relationship with $\Delta h$ as the steady-state data. This suggests that the mean curvature of the potential energy density function averaged over all instantaneously occupied basins in steady flow has the same dependence on potential energy as the curvature within a single megabasin

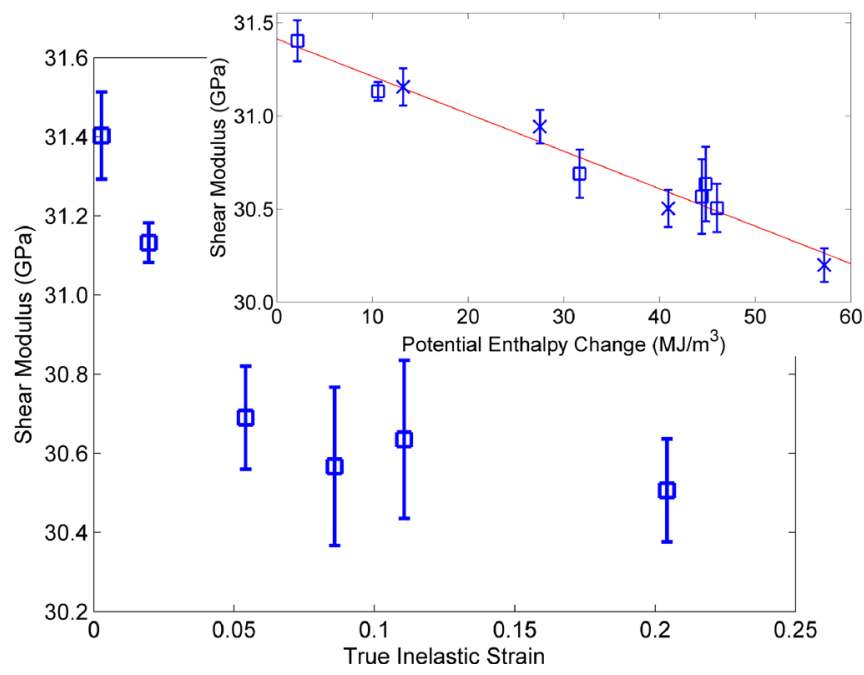

FIG. 4 (color online). Ultrasonic measurements of the highfrequency shear modulus $G$ plotted against the measured inelastic strain. Inset: Transient $G$ vs $\Delta h$ data gathered in the present study ( $\square$ ), together with steady-state $G$ vs $\Delta h$ data gathered previously $(\times)$ [15]. (Note that the $\Delta h$ datum in Ref. [15] was shifted by $33 \mathrm{MJ} / \mathrm{m}^{3}$ to coincide with the datum in the present study.) The line represents a linear regression to all data.

in transient deformation. This result provides support for rheological laws based on averages over instantaneously occupied megabasins during steady flow [9-11].

In Fig. 5, we plot the anelastic strain recovery against the corresponding inelastic strain. During the initial stages of transient deformation, the system exhibits complete strain recovery and thus retains full memory of its initial configurational state. As the transient deformation advances, this memory gradually breaks down until it is completely lost at steady state, where deformation becomes completely irreversible. This gradual breakdown of the system's memory suggests a smooth anelastic to plastic transition. Interestingly, a plot of $G$ against the anelastic strain recovery (inset in Fig. 5) gives an approximately linear relationship. A linear relation between $G$ and the fraction of anelastic strain recovery suggests that $G$ is indeed the governing thermodynamic state variable for the flow transition, as previously proposed [9].

We therefore find the anelastic to plastic transition to conform well to the proposed "subbasin-megabasin" organization of the landscape [7]. We now explore the relationship of this transition to the concept of elastic matrix confinement [4] adopted to explain the anelasticity of STZ's [3]. We have demonstrated that reversible anelasticity does not completely break down until the system attains enough configurational energy to surmount barriers between megabasins. We now argue that the onset of irreversible plasticity coincides with the collapse of the STZmatrix elastic confinement. The critical shear stress measured in the present experiments is $\tau_{c}=\sigma_{c} / 2 \approx 500 \mathrm{MPa}$ $\left(\sigma_{c} \approx 1000 \mathrm{MPa}\right.$ is the measured uniaxial peak stress shown in Fig. 2). We demonstrate here that $\tau_{c}$ is roughly 


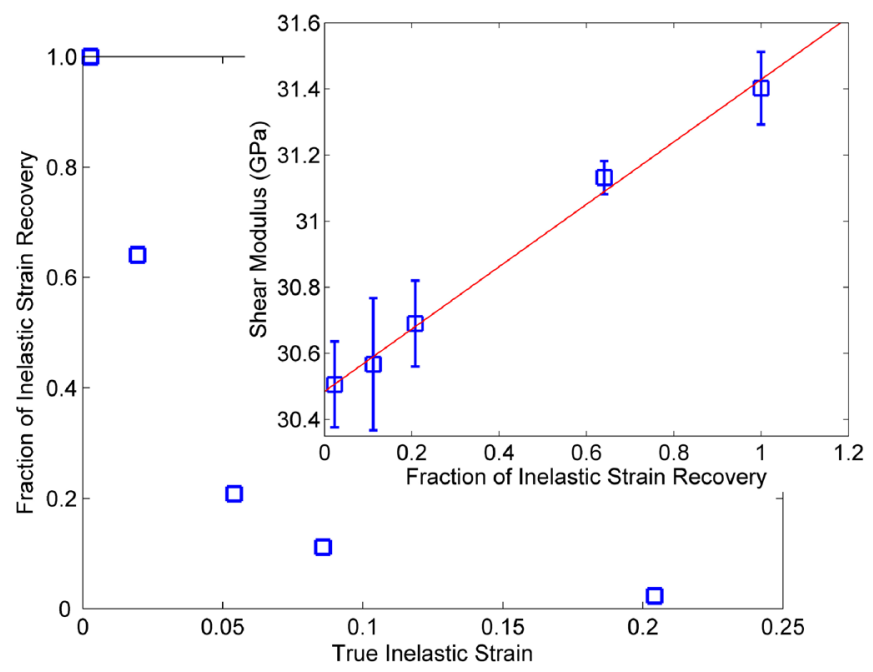

FIG. 5 (color online). Fraction of anelastic strain recovery plotted against the inelastic strain. (Note that no data were produced at 0.1106 strain due to specimen mishandling.) Inset: $G$ plotted against the fraction of anelastic strain recovery. The line represents a linear regression to the data.

equal to an effective "backstress" determined by the slope of the average potential energy density with respect to the average inelastic shear strain (as illustrated in Fig. 1). We interpret this slope as the "confinement stress" operating on the plastic STZ core. Transforming the uniaxial strain in Fig. 3 to a shear strain $\gamma$ (using Poisson's ratio $\nu$ at $T_{g}$ of $\sim 0.4$ [14]), the slope of $\Delta h$ with respect to $\gamma$ is evaluated to be $d \Delta h / d \gamma \approx 420 \mathrm{MPa}$, very close to $\tau_{c}$ measured in the mechanical tests. We interpret the critical stress associated with the anelastic to plastic transition as the effective Eshelby backstress. For the present conditions (548 K; $1 \times$ $\left.10^{-4} \mathrm{~s}^{-1}\right), \tau_{c}=400-500 \mathrm{MPa}$.

In conclusion, by probing the instantaneous changes in stored potential energy, isoconfigurational shear modulus, and anelastic strain recovery, we investigated the transition from anelastic to plastic response in a transiently deforming metallic glass-forming liquid. The data revealed that the transition can be separated into reversible and irreversible configurational hopping across the liquid energy landscape, identified with the $\beta$ and $\alpha$ relaxation processes, respectively. A critical stress arising from the transition has been recognized as an effective Eshelby backstress confining the STZ's to be elastically coherent with the surrounding matrix. These observations provide a direct link between the apparent anelastic to plastic transition and the collapse of the STZ-matrix coherency condition. More importantly, these observations suggest that the subbasin-megabasin organization of the potential energy landscape is a direct consequence of the STZ confinement stress and the associated memory effect.

This work was supported in part by the MRSEC Program of the National Science Foundation under Grant
No. DMR-0520565 and in part by the Leibniz Program under Grant No. SFB602. The authors are grateful to G. Ravichandran for providing the loading apparatus.

*To whom all correspondence should be addressed. marios@caltech.edu

[1] A. S. Argon, Acta Metall. 27, 47 (1979).

[2] A.S. Argon and H. Y. Kuo, Mater. Sci. Eng. 39, 101 (1979).

[3] A. S. Argon and H. Y. Kuo, J. Non-Cryst. Solids 37, 241 (1980); A. S. Argon and L. T. Shi, Acta Metall. 31, 499 (1983); L. T. Shi, A. S. Argon, and H. Y. Kuo, Scr. Metall. 17, 1015 (1983).

[4] J. D. Eshelby, Proc. R. Soc. A 241, 376 (1957); Proc. R. Soc. A 252, 1271 (1959).

[5] M. D. Demetriou, J. C. Hanan, C. Veazey, M. Di Michiel, N. Lenoir, E. Ustundag, and W. L. Johnson, Adv. Mater. 19, 1957 (2007).

[6] G. P. Johari and M. Goldstein, J. Chem. Phys. 53, 2372 (1970).

[7] P. G. Debenedetti and F. H. Stillinger, Nature (London) 410, 259 (2001).

[8] W. L. Johnson and K. Samwer, Phys. Rev. Lett. 95, 195501 (2005).

[9] M.D. Demetriou, J.S. Harmon, M. Tao, G. Duan, K. Samwer, and W. L. Johnson, Phys. Rev. Lett. 97, 065502 (2006).

[10] J. S. Harmon, M. D. Demetriou, and W. L. Johnson, Appl. Phys. Lett. 90, 171923 (2007).

[11] W.L. Johnson, M. D. Demetriou, J. S. Harmon, M. L. Lind, and K. Samwer, MRS Bull. 32, 644 (2007).

[12] J. Lu, G. Ravichandran, and W. L. Johnson, Acta Mater. 51, 3429 (2003).

[13] M. L. Lind, G. Duan, and W. L. Johnson, Phys. Rev. Lett. 97, 015501 (2006).

[14] N. Nishiyama, A. Inoue, and J. Z. Jiang, Appl. Phys. Lett. 78, 1985 (2001).

[15] J.S. Harmon, M.D. Demetriou, M. Tao, and W.L. Johnson, Appl. Phys. Lett. 90, 131912 (2007).

[16] The deformed specimens were annealed at $558 \mathrm{~K}$ for four hours and subsequently requenched. The anelastic strain recovery during annealing was evaluated by measuring the change in specimen heights before and after annealing. Calipers with an accuracy of $\pm 0.005 \mathrm{~mm}$ were used. Additional annealing showed no measurable strain recovery, confirming that the annealing duration was sufficient to determine the maximum recoverable strain. The reference specimen (that was preannealed and relaxed at $548 \mathrm{~K}$ ) showed no measurable height change after annealing at $558 \mathrm{~K}$ for four hours. The amorphous nature of the quenched specimens after annealing was confirmed by x-ray diffraction.

[17] G. Duan, M. L. Lind, M.D. Demetriou, W. L. Johnson, W. A. Goddard III, T. Cagin, and K. Samwer, Appl. Phys. Lett. 89, 151901 (2006); M. Zink, K. Samwer, W. L. Johnson, and S. G. Mayr, Phys. Rev. B 74, 012201 (2006). 